EPJ Web of Conferences 82, 01011 (2015)

DOI: $10.1051 /$ epjconf/20158201011

(C) Owned by the authors, published by EDP Sciences, 2015

\title{
Operation reliability analysis of independent power plants of gas-transmission system distant production facilities
}

\author{
Maksim V. Piskunov, Ivan S. Voytkov, Olga V. Vysokomornaya a and Vladimir S. Vysokomorny \\ National Research Tomsk Polytechnic University, 634050 Tomsk, Russia
}

\begin{abstract}
The new approach was developed to analyze the failure causes in operation of linear facilities independent power supply sources (mini-CHP-plants) of gas-transmission system in Eastern part of Russia. Triggering conditions of ceiling operation substance temperature at condenser output were determined with mathematical simulation use of unsteady heat and mass transfer processes in condenser of mini-CHP-plants. Under these conditions the failure probability in operation of independent power supply sources is increased. Influence of environmental factors (in particular, ambient temperature) as well as output electric capability values of power plant on mini-CHP-plant operation reliability was analyzed. Values of mean time to failure and power plant failure density during operation in different regions of Eastern Siberia and Far East of Russia were received with use of numerical simulation results of heat and mass transfer processes at operation substance condensation.
\end{abstract}

\section{Introduction}

Powering is an important aspect of any production. Stable supply necessary for the implementation of technological processes of energy determines the stability of the enterprises. Questions of reliability and efficiency of electric utility are particularly important for businesses with remote objects on its balance sheet from a centralized power system (such as gas and oil pipelines of large extent). Typically, the functioning of these objects is accompanied by the need to accommodate small linear features, such as devices of electrochemical protection, crane components, stations of teleautomatic system, etc. If gas or oil pipelines are located in areas remote from the centralized power system, the only possible way of organizing powering is the use of independent sources of powering.

For powering gas pipelines in the Far East of Russia, independent energy sources are used with a closed thermodynamic cycle of power up to $4 \mathrm{~kW}$ (Fig. 1). Such sources operate on natural gas and are characterized by a considerable duration of automatic operation and a high value of thermal efficiency. Nevertheless, often there are failures of this equipment which negatively affect functioning of the gas transmission system. Thus the analysis of statistical data showed [1] that one of basic reasons of violations of operation of a power source is appearance of extremely high value of temperature of working substance on an output from the condenser.

\footnotetext{
${ }^{a}$ Corresponding author: vysokomornaja@tpu.ru
}

This is an Open Access article distributed under the terms of the Creative Commons Attribution License 4.0, which permits unrestricted use, distribution, and reproduction in any medium, provided the original work is properly cited. 


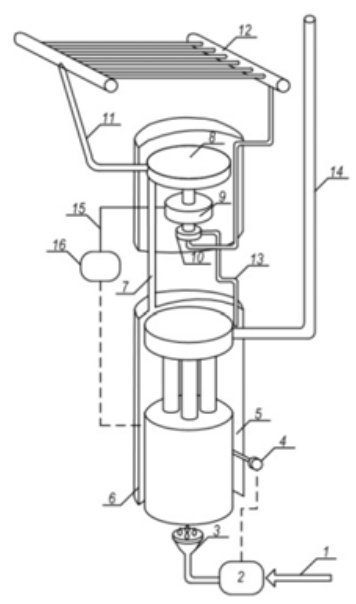

Figure 1. Schematic illustration of electric power installation using the closed cycle of steam: 1 - fuel inlet; 2 fuel supply panel; 3 - fuel burner; 4 - thermostat; 5 - organic liquid; 6 - steam generator; 7 - steam; 8 - turbine; 9 - AC generator; 10 - pump; 11 - steam supply in a condenser; 12 - condenser; 13 - condensed fluid outlet; 14 exhaust stack; 15 - input cables of alternating current to the rectifier; 16 - electric service panel.

The purpose of article - the analysis of operation modes of independent power installations which cause failures because of extremely high temperature in the condenser, and also time between failures determination taking into account climatic conditions of the Far East of Russia.

\section{Problem statement}

Power installation (Fig. 1) functions by the following principle. The torch 3 working at natural gas, in the steam generator 6 heats, and then evaporates organic working substance (dichlorobenzene). The working substance in a vaporous status moves in the turbine 8 and brings into rotation a shaft of a turbogenerator of an alternating current 9 . Further the working substance arrives in the condenser with the air cooling 12 , consisting of 2 ranks of ribbed tubes the internal diameter of $D_{\text {in }}=38 \mathrm{~mm}$ united by collectors. The working substance is condensed and with pump 10 comes back to the steam generator 6 , and the cycle becomes isolated. It should be noted that dichlorobenzene besides using in a running cycle of power installation is also the liquid greasing bearings of sliding of a shaft of a turbogenerator.

It was supposed that dichlorobenzene in a vaporous state at a temperature of saturation $T_{S}$ arrives in tubes of the condenser (Fig. 2). Through surfaces of tubes of the condenser the membrane whose thickness in the process of a current of vapor-liquid mix in the channel increases is formed. Process of condensation comes to the end at achievement of a share of steam in vapor-liquid mix of working substance of the standard value declared by manufacturer [2].

\section{Mathematical model}

The non-stationary differential equations describing process of condensation of a working body in tubes of condenser installation (Fig. 2) and corresponding formulated physical problem definition, have the following appearance. 


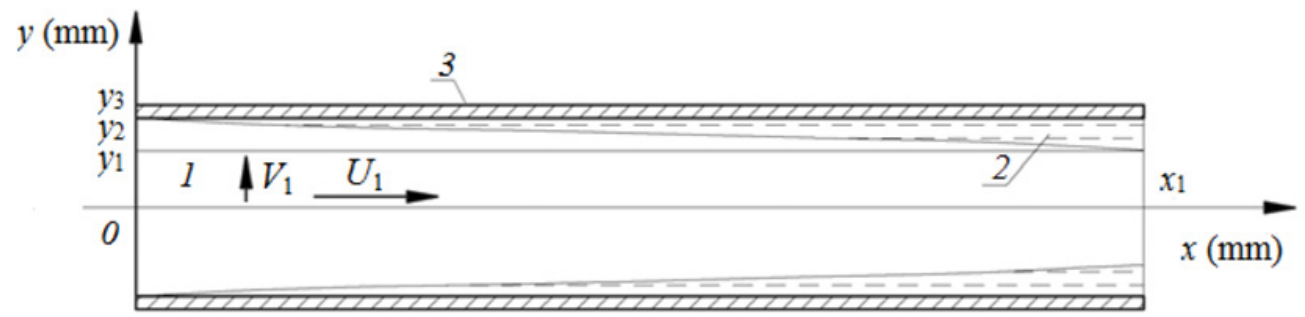

Figure 2. Schematic representation of solution region for the problem: 1 - vapors of the working substance; 2 condensed fluid; 3 - wall of the condenser pipe; 4 - surrounding air.

The heat conductivity equation for working substance in a condensation zone $\left(T_{1}=T_{s}, 0<x<x_{1}\right.$, $\left.0<y<y_{1}\right)$ :

$$
\frac{\partial T_{1}}{\partial t}+U_{1} \frac{\partial T_{1}}{\partial x}+V_{1} \frac{\partial T_{1}}{\partial y}=a_{1}\left(\frac{\partial^{2} T_{1}}{\partial x^{2}}+\frac{\partial^{2} T_{1}}{\partial y^{2}}\right) .
$$

The heat conductivity equation for working substance out of a condensation zone $\left(T_{1}<T_{s}, 0<x<x_{1}\right.$, $\left.y_{1}<y<y_{2}\right)$ :

$$
\frac{\partial T_{2}}{\partial t}=a_{2}\left(\frac{\partial^{2} T_{2}}{\partial x^{2}}+\frac{\partial^{2} T_{2}}{\partial y^{2}}\right)
$$

The heat conductivity equation for a wall $\left(0<x<x, y_{2}<y<y_{3}\right)$ :

$$
\frac{\partial T_{3}}{\partial t}=a_{3}\left(\frac{\partial^{2} T_{3}}{\partial x^{2}}+\frac{\partial^{2} T_{3}}{\partial y^{2}}\right) .
$$

Equation of diffusion of vapors of working substance $\left(0<x<x_{1}, 0<y<y_{1}\right)$ :

$$
\frac{\partial C_{\mathrm{V}}}{\partial t}+U_{1} \frac{\partial C_{\mathrm{V}}}{\partial x}+V_{1} \frac{\partial C_{\mathrm{V}}}{\partial y}=D_{1}\left(\frac{\partial^{2} C_{\mathrm{V}}}{\partial x^{2}}+\frac{\partial^{2} C_{\mathrm{V}}}{\partial y^{2}}\right) .
$$

Equation of movement of vapors of working substance $\left(0<x<x_{1}, 0<y<y_{1}\right)$ :

$$
\rho_{1} U_{1} \frac{\partial U_{1}}{\partial x}+\rho_{1} V_{1} \frac{\partial U_{1}}{\partial y}=-\frac{\partial P_{1}}{\partial x}+\mu_{1} \frac{\partial^{2} U_{1}}{\partial y^{2}}
$$

Equation of continuity of vapors of working substance $\left(0<x<x_{1}, 0<y<y_{1}\right)$ :

$$
\frac{\partial \rho_{1} U_{1}}{\partial x}+\frac{\partial \rho_{1} V_{1}}{\partial y}=0
$$

Equation of a condition of vapors of working substance $\left(0<x<x_{1}, 0<y<y_{1}\right)$ :

$$
P_{1}(x, y)=\frac{\rho_{1}(x, y)}{M_{1}} \cdot R_{\mathrm{t}} \cdot T_{1}(x, y) .
$$

$T$ - temperature (K); $t$ - time (s); $x, y$ - coordinates of the Cartesian system of coordinates $(\mathrm{mm}) ; U, V-$ speed components in a projection to axes $x$ and $y(\mathrm{~m} / \mathrm{s}) ; a$ - coefficient of heat diffusivity $\left(\mathrm{m}^{2} / \mathrm{s}\right) ; C_{\mathrm{v}}$ - molar concentration $\left(\mathrm{mol} / \mathrm{m}^{3}\right) ; D$ - coefficient of diffusion $\left(\mathrm{m}^{2} / \mathrm{s}\right) ; \rho$ - density $\left(\mathrm{kg} / \mathrm{m}^{3}\right) ; P$ - pressure $\left(\mathrm{N} / \mathrm{m}^{2}\right) ; \mu$ - coefficient of dynamic viscosity $\left(\mathrm{kg} /(\mathrm{m} \cdot \mathrm{s}) ; M-\right.$ molar weight $(\mathrm{kg} / \mathrm{mol}) ; R_{t}-$ the gas constant $(\mathrm{J} /(\mathrm{mol} \cdot \mathrm{K})) ; T_{0}$ - reference temperature $(\mathrm{K}) ; \lambda$ - coefficient of heat conductivity $(\mathrm{W} /(\mathrm{m} \cdot \mathrm{K}))$; $Q_{c}$ - hidden energy of phase transition $(\mathrm{J} / \mathrm{kg}) ; W_{c}$ - condensation speed $\left(\mathrm{kg} /\left(\mathrm{m}^{2} \cdot \mathrm{s}\right)\right) ; \alpha$ - thermolysis coefficient, $\left(\mathrm{W} /\left(\mathrm{m}^{2} \cdot \mathrm{K}\right)\right) ; T_{\text {out }}$ - ambient temperature $(\mathrm{K}) ; T_{\text {input }}-$ temperature of vapors on an entrance to the channel $(\mathrm{K}) ; U_{0}, V_{0}$ - initial distributions of speed $(\mathrm{m} / \mathrm{s}) ; C_{0}-$ concentration of steam on an 


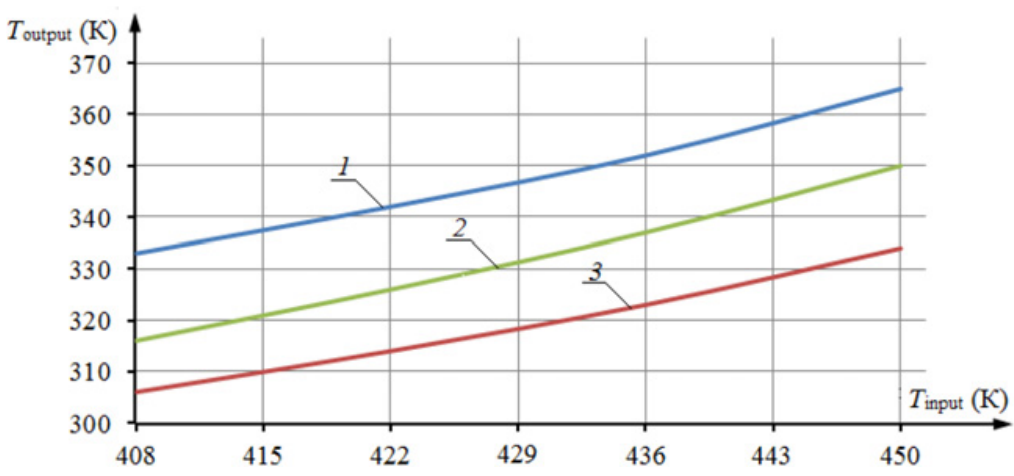

Figure 3. Values of output temperature of actuation medium $T_{\text {output }}$ at various temperatures of dichlorobenzene on an entrance to the condenser $T_{\text {input }}: 1-T_{\text {out }}=303 \mathrm{~K} ; 2-T_{\text {out }}=273 \mathrm{~K} ; 3-T_{\text {out }}=253 \mathrm{~K}$.

entrance to the channel $\left(C_{0}=\rho_{\text {linput }} / M_{1}\right)\left(\mathrm{mol} / \mathrm{m}^{3}\right) ; \rho_{\text {linput }}-$ density of vapor on an entrance to the channel $\left(\mathrm{kg} / \mathrm{m}^{3}\right) ; \beta$ - the nondimensional coefficient of condensation $(\beta=0,1) ; \mathrm{k}_{\beta}$ - padding coefficient equa 0,$4 ; P^{n}$ - pressure of saturated steam $\left(\mathrm{N} / \mathrm{m}^{2}\right)$; the indexes " 1 ", " 2 ", " 3 " correspond to vapors of dichlorobenzene, its liquid phase and a material of pipes of the condenser. The regional conditions corresponding to chosen statement, are described in.

The system of nonstationary differential equations with the corresponding boundary conditions (1)-(8) was solved by a method of finite differences [3, 4]. Difference analogs of differential equations (1)-(8) were solved by a local and one-dimensional method. The sweep method was applied to the solution of one-dimensional difference equations with the use of the implicit four-pointwise scheme. The method of prime iterations was applied to the solution of the non-linear equations. The technique of an assessment of reliability of results of the executed numerical researches is based on the checking of conservatism of the applied difference scheme.

\section{Results and discussion}

Numerical researches are performed at typical values of parameters works of considered condenser installations of independent sources of power supply (Fig. 2): reference temperature of actuation medium on an entrance to the condenser $T_{1}=403 \mathrm{~K}$; heat effect of condensation of $Q_{c}=311.7 \mathrm{~kJ} / \mathrm{kg}$; sizes of area of the decision $H_{x}=400 \mathrm{~mm}, H_{y}=1500 \mathrm{~mm}$; molecular mass dichlorobenzene $M=$ $147 \mathrm{~kg} / \mathrm{kmol}$; the nondimensional coefficient of evaporation $\beta=0.1$; speed of a working body in the condenser $V_{1}=0.01 \mathrm{~m} / \mathrm{s}$; heat-transfer coefficient at condensation of vapors of dichlorobenzene in the condenser channel $\alpha_{1-2}=650 \mathrm{~W} /\left(\mathrm{m}^{2} \cdot \mathrm{K}\right)$.

Thermal characteristics of dichlorobenzene, steel, air are provided in [5-7].

Thus critical value of temperature of dichlorobenzene on condenser escaping at which excess the risk of emergence of failure of an independent source of power supply considerably increases, $T_{\text {output }} \approx 340 \mathrm{~K}$. Thus, on the basis of the received results of numerical model operation it is possible to draw a conclusion that at a temperature of a free air $T_{\text {out }}=303 \mathrm{~K}$ critical value of temperature of actuation medium after the condenser is already possible at an entrance temperature $T_{\text {input }} \approx 420 \mathrm{~K}$, that corresponds to power $P=1500 \mathrm{~W}$ (less than $40 \%$ of capacity).

The dependence of temperature of a working body on condenser escaping from temperature of air environmental the condenser is defined at various duties (Table). The received dependences well correspond to the data presented in Fig. 3. 
Table 1. Dependence of temperature of dichlorobenzene on condenser escaping from temperature of the ambient air.

\begin{tabular}{|c|c|c|c|c|c|c|c|}
\hline $\mathrm{T}_{\text {out }}(\mathrm{K})$ & 253 & 263 & 273 & 283 & 293 & 303 & 313 \\
\hline \multicolumn{8}{|c|}{ At a temperature on an entrance to the condenser $\mathrm{T}_{\text {input }}=403 \mathrm{~K}$} \\
\hline $\mathrm{T}_{\text {output }}(\mathrm{K})$ & 306 & 309 & 313 & 317 & 323 & 330 & 337 \\
\hline \multicolumn{8}{|c|}{ At a temperature on an entrance to the condenser $\mathrm{T}_{\text {input }}=426 \mathrm{~K}$} \\
\hline $\mathrm{T}_{\text {output }}(\mathrm{K})$ & 314 & 318 & 322 & 326 & 332 & 338 & 346 \\
\hline \multicolumn{8}{|c|}{ At a temperature on an entrance to the condenser $\mathrm{T}_{\text {input }}=447 \mathrm{~K}$} \\
\hline $\mathrm{T}_{\text {output }}(\mathrm{K})$ & 334 & 338 & 344 & 348 & 354 & 362 & 369 \\
\hline
\end{tabular}

Received at application of the developed mathematical model results of numerical researches allow to define a time between failures of considered independent sources of power supply at operation specific climatic conditions. As an example Russia can consider the Amur region, on the territory of which the extent of the main gas pipelines makes more than $1200 \mathrm{~km}$. The data presented in Fig. 3 and in Table allow to draw a conclusion that when functioning independent power installation at a rated power $\left(P=2000 \mathrm{~W}, T_{\text {input }}=426 \mathrm{~K}\right)$ temperature of actuation medium on escaping of the condenser will exceed critical value at an ambient temperature $T_{\text {out }}=305 \mathrm{~K}$. According to long-term climatic supervision [8] in the Amur region duration of weather conditions within a year with air temperature $305 \mathrm{~K}$ and higher makes $840 \mathrm{~h}$. Thus, time per fault at the capacity $P=2000 \mathrm{~W}$ in climatic conditions of the Amur region will make $T_{0}=7920 \mathrm{~h}$.

It should be noted that climatic conditions considered as an example for calculation of time per fault of the region are characterized by the big duration of the periods with the under air temperatures. In case of use of similar independent power installations in regions of the central part of Russia or in the countries of Europe with warmer climate and larger duration of the periods of time with elevated temperature of air indexes of reliability of work of an independent power source will be considerably lowered.

\section{Conclusions}

The mathematical model, allowing to predict emergence of failures of an independent source of the power supply functioning on a closed thermodynamic cycle, during the work in the wide range of a output capacity and in various climatic conditions is developed.

As a result of the conducted numerical researches of condensation process of actuation medium (dichlorobenzene) in an air capacitor of the independent power installation working on a closed thermodynamic cycle, dependences of output temperature of actuation medium on its reference temperature and on temperature of an ambient air are received. Emergency conditions of operation at which emergence of failures of power installation for the reason of extremely high temperature on condenser escaping is possible are defined.

This work was financially supported by the Ministry of Education and Science of the Russian Federation (contract 2.1321.2014) and subsidy within implementation of the Program of competitiveness increase of National research Tomsk polytechnic university. 


\section{References}

[1] O.V. Vysokomornaya, V.S. Vysokomorny, P.A. Strizhak, Energetic 8, 31 (2013) [in Russian]

[2] EPS Operation and Maintenance Manual (powered by CCVT) (ORMAT SYSTEMS LTD, Yavne, 2006)

[3] D.O. Glushkov, G.V. Kuznetsov, P.A. Strizhak, Russ. J. Phys. Chem. B 8, 196 (2014)

[4] D.O. Glushkov, P.A. Strizhak, Adv. Mech. Eng. 2014, 269321 (2014)

[5] Yu.M. Babikov, Organic and Organosilicone Heat Conductors (Energia, Moscow, 1975) [in Russian]

[6] N.B. Vargaftik, Tables of Thermophysical Properties of Liquids and Gases (Hemisphere Publishing, New York, 1975)

[7] Liquid Materials Handbook, The Atomic Energy Commission (Department of the Navy, Washington DC, 1952)

[8] Building climatology, Construction norms and rules of the Russian Federation 23-01-99 (Standartinform, Moscow, 1999) [in Russian] 